\title{
Li-Tm (Lithium-Thulium)
}

\section{H. Okamoto}

The Li-Tm phase diagram was unknown in [Massalski2].

Figure 1 shows the Li-Tm phase diagram constructed on the basis of a schematic phase diagram and a table of invariant reaction temperatures and compositions given in [1998Gan].

Table 1 shows Li-Tm crystal structure data.

\section{Reference}

1998Gan: N.N. Ganiev, Kh.M. Nazarov, and M.D. Badalov, Interaction of Lithium with Rare-Earth Metals, Izv. Ross. Akad. Nauk, Metally, 1998, (6), p 109-112, in Russian; TR: Russ. Metall., 1998, (6), p 132-136

Table 1 Li-Tm crystal structure data

\begin{tabular}{|c|c|c|c|c|c|}
\hline Phase & Composition, at.\% Tm & Pearson symbol & Space group & Strukturbericht designation & Prototype \\
\hline$(\beta \mathrm{Li})$ & $0-5.88$ & $c I 2$ & $\operatorname{Im} \overline{3} m$ & $A 2$ & $\mathrm{~W}$ \\
\hline$(\mathrm{Tm})$ & ? to 100 & $h P 2$ & $\mathrm{P6}_{3} / m m c$ & $A 3$ & $\mathrm{Mg}$ \\
\hline
\end{tabular}



Fig. 1 Li-Tm phase diagram 